\title{
Toxicidad del cobre en larvas nauplii del camarón comercial Artemesia longinaris Bate (Crustacea, Decapoda, Penaeidae)*
}

\author{
Marcelo A. Scelzo \\ Departamento de Ciencias Marinas, Facultad de Ciencias Exactas y Naturales \\ Universidad Nacional de Mar del Plata \\ Funes 3350, 7600 Mar del Plata, Argentina
}

RESUMEN. Se evaluó la toxicidad del cobre, empleando sulfato de cobre en larvas nauplii sin alimentar del camarón comercial Artemesia longinaris mediante bioensayos de 72 horas de duración, con el objeto de determinar la dosis efectiva media o concentración letal (LC50) y grado de desarrollo (estadio larval). Se prepararon concentraciones de cobre equivalentes a 1000, 500, 250 y 125 partes por billón (ppb) en agua de mar (salinidad de $34 \%$ ). Un total de 90 larvas nauplii por tratamiento (tres réplicas de 30 larvas c/u) fueron acondicionadas en recipientes de vidrio con 300$500 \mathrm{ml}$ de cada solución. Como control se empleó agua de mar con EDTA. Los resultados del test de toxicidad aguda mostraron una relación directa entre mortalidad y concentración de cobre. A una concentración de 1000 ppb, los nauplii mueren en menos de $24 \mathrm{hr}$. Los valores de LC50 para los diferentes períodos de los restantes tratamientos según el método de probits fueron: 660 ppb para 24 hr, 378,5 ppb para 48 hr y 212,3 ppb para 72 hr. La muda (crecimiento) de las larvas fue inhibida a concentraciones superiores a $125 \mathrm{ppb}$. En el «control», las larvas mudaron a protozoea a partir de las $72 \mathrm{hr}$ y todas las larvas se encontraron en este estadio a las $96 \mathrm{hr}$.

Palabras claves: contaminación, bioensayos, toxicidad, $\mathrm{Cu}$, crustáceos, camarón peneido Artemesia longinaris, nauplius.

\section{Copper toxicity in nauplii larvae of the commercial shrimp Artemesia longinaris (Crustacea, Decapoda, Penaeidae)*}

\begin{abstract}
Copper toxicity in the nauplius larvae of the penaeid shrimp Artemesia longinaris was evaluated through biossays, to determine the lethal concentration (LC50) and stage of development (larval stage). A solution of copper sulphate in sea water (salinity $34 \%$ ) at concentrations equivalent to 1000, 500, 250 and 125 ppb of copper was utilized. A total of 90 nauplii per treatment (three replicates of 30 larvae each) were conditioned in glass bowls containing 300-500 ml of solution. Pure sea water with EDTA added was utilized as «control». The biossays lasted 72 hrs. and the nauplii larvae were not fed. Results of the acute toxicity test show a direct relationship between mortality and copper concentrations. At $1000 \mathrm{ppb}$ the nauplii died during the first $24 \mathrm{hr}$. The LC50 values (probits analysis) were: $660.9 \mathrm{ppb}$ for $24 \mathrm{hr}, 378.5$ for $48 \mathrm{hr}$, and $212.3 \mathrm{ppb}$ for $72 \mathrm{hr}$. Molting (growth) of the larva was inhibited at concentrations higher than $125 \mathrm{ppb}$. In the controls, the protozoeae stage appeared at $72 \mathrm{hr}$ and all larvae were found at that stage at the $96 \mathrm{hr}$ of treatment.
\end{abstract}

Key words: pollution, biossays, toxicity, $\mathrm{Cu}$, crustacean, penaeid shrimp, nauplius, Artemesia longinaris.

\section{INTRODUCCION}

El gran desarrollo de los procesos de industrialización del mundo moderno trae como consecuencia la liberación de desechos que se incorporan al ecosistema, muchos de ellos son considerados altamente tóxicos para los organismos acuáticos, como es el caso de los iones de los metales pesados, los cuales son un grupo de elementos químicos biológicamente activos y de baja abundancia en las aguas naturales. Algunos metales traza, como hierro, manganeso, zinc, cobre y cobalto, son nutrientes

*Contrtibución del Departamento de Ciencias Marinas №86 
esenciales que sirven como co-factores de numerosas enzimas, pero el cobre, cobalto y zinc son también tóxicos en altas concentraciones (Department of Energy, USA, 1987). Todos los metales, incluyendo los micronutrientes esenciales, son virtualmente tóxicos para los organismos acuáticos y para los seres humanos si los niveles de exposición son suficientemente altos (Laws, 1981). El cobre se halla en forma natural en el agua de mar en concentraciones bajas, alrededor de 2 microgramos por litro o partes por billón. En el agua de los ríos, la concentración es algo mayor, aproximadamente 7 microgramos por litro. A través del proceso de concentración biológica, el cobre es bioacumulado por los organismos vivos filtradores, alcanzando una concentración de varios órdenes de magnitud en los macroinvertebrados (Waldichuk, 1974; Laws, 1981). La asimilación implica la formación de complejos con sustancias orgánicas, no siendo fácilmente excretados.

La mayor concentración de cobre en los crustáceos decápodos se encuentra en el hepatopáncreas y en la sangre, donde forma parte del pigmento respiratorio o hemocianina (Bryan, 1968). El cobre es un contaminante metálico marino que tiene gran importancia debido a que la aleación cobre-níquel se emplea como antifouling en los intercambiadores de calor del sistema de enfriamiento con agua de mar de las plantas atómicas (Department of Energy, 1987). Las sales de muchos metales pesados poseen ciertas propiedades biocidas (Waldichuk, 1974). El sulfato de cobre, es un poderoso alguicida, siendo parte integrante del producto comercial «Copper Control» Argen Chemical Laboratory, USA, de uso generalizado en acuacultura, tanto para el control de la vegetación acuática indeseable como en la inducción de la muda en los camarones y langostinos adultos. La toxicidad aguda del cobre difiere grandemente de acuerdo al nivel trófico del organismo y al estadio de su ciclo de vida (Department of Energy, 1987).

Aunque los organismos vivos requieren de ciertas cantidades de iones metálicos para realizar eficazmente los procesos fisiológicos, un exceso de iones de metales pesados causan estrés que se manifiesta en forma subletal o causan la muerte. Los organismos marinos han desarrollado un número variado de estrategias adaptativas que son denominadas colectivamente como "metabolismo de los metales" e incluyen la regulación activa de la absorción del metal, la distribución, el metabolismo y la excreción (Department of Energy, 1987). Los estudios recientes han demostrado que la toxicidad del cobre está mas directamente relacionada a la actividad de los iones libres del cobre y su especificidad química que a la concentración total del metal, o bien, a las combinaciones de diferentes concentraciones de otros metales (Costlow y Sanders, 1987). Sin embargo, la toxicidad del cobre puede ser disminuida por la presencia o adición de sustancias quelantes, tanto naturales como sintéticas (Department of Energy, 1987).

El camarón Artemesia longinaris (Penaeidae) y el langostino Pleoticus muelleri (Solenoceridae), son dos crustáceos marinos explotados comercialmente en Argentina, siendo la segunda especie destinada a la exportación. Ambas especies están siendo sometidas a experiencias de acuicultura tanto en aguas de la Provincia de Buenos Aires como en la región patagónica (Boschi y Scelzo, 1978; Scelzo, 1987; Iorio et al., 1990). Los estudios sobre toxicidad cobran mayor relevancia cuando son llevados a cabo sobre especies de importancia en la pesca y fundamentalmente en la acuicultura, donde es necesario el control de la calidad del agua en las instalaciones, especialmente para la cría de larvas. Los estadios tempranos del desarrollo de los organismos acuáticos (huevos y larvas) pueden ser usados como medida general de estrés debido a que representan un estado muy sensible del ciclo de vida de los organismos por ser un período de rápido crecimiento y división celular (Department of Energy, 1987; Crecelius y Bloom, 1987). Este trabajo tiene como objetivo realizar un test de toxicidad aguda del cobre, en solución como sulfato de cobre, sobre el primer estadio larval (nauplius) del camarón $A$. longinaris.

\section{MATERIAL Y METODOS}

Los nauplii del camarón A. longinaris se obtuvieron mediante desoves en laboratorio provenientes de hembras capturadas en el medio ambiente. Luego, se preparó una solución stock de CuSO . A través de un test exploratorio, se determinó una concentración claramente letal y se diluyó a concentraciones de cobre equivalentes a 1000, 500, 250 y 125 ppb en agua de mar filtrada, con salinidad 33-34\%o y temperaturas entre $18-22^{\circ} \mathrm{C}$. Como «control»se empleó agua de mar con EDTA al 1\% (Boschi y Scelzo, 1978; Scelzo, en prensa).

Se realizaron ensayos de toxicidad aguda con un total de 450 larvas, en estadio de nauplius de primer día de eclosión provenientes de distintas 
hembras. Ellas fueron distribuidas en 90 individuos para cada uno de los diferentes tratamientos, mas el experimento de referencia (Tabla 1). Cada tratamiento consistió en tres grupos (réplicas) de 30 larvas cada uno. Las larvas de cada réplica fueron acondicionadas en recipientes de vidrio con 300-500 ml. Dado que en el estadio de nauplius, las larvas son lecitotróficas y se nutren a expensas del vitelo intracelular, no se les suministró alimento (Boschi y Scelzo, 1978).

Los ensayos de toxicidad aguda fueron estáticos, sin aireación, con recambio total de la solución al cabo de 24 hrs. Diariamente, el contenido de cada recipiente fue filtrado por un tamiz con abertura de malla de 77 micrones. Las larvas vivas y muertas fueron observadas bajo microscopio estereoscópico. Ello permitió llevar un registro diario de mortalidad, crecimiento (mudas) y desarrollo de setas, para cada tratamiento.

Los resultados de toxicidad aguda fueron expresados en términos de mortalidad, número total y porcentajes (Tabla 1), al igual que efectos de comportamiento como: muda, estadio, desarrollo de setas, etc., según lo recomendado por la Water Pollution Control Federation (1980). Los valores de toxicidad se indican como concentración letal media (LC50), es decir, la concentración letal que afecta al 50\% de la población y organismos bajo estudio, conjuntamente con los valores de los límites de confianza respectivos (95\% y 99\%) (Tabla 2). En el análisis de datos, se empleó el método de Probit (Litchfield y Wilcoxon, 1949; Sprague, 1969), utilizando las técnicas de cálculo mediante programas en computadoras.

\section{RESULTADOS}

En la Tabla 1 se indican los resultados de toxicidad en términos de mortalidad de los nauplii del camarón A. longinaris sometidos a diferentes concentraciones de cobre. Existe una relación directa entre la concentración de cobre y la mortalidad de las larvas para los diferentes tiempos de exposición (Fig. 1). En una concentración de cobre equivalente a 1000 ppb, se produce la mortalidad de todas las larvas durante las primeras $24 \mathrm{hr}$ de experimentación, mientras que se obtiene mortalidad creciente en función de las restantes concentraciones. En 500 ppb de cobre, la mortalidad total se produce luego de las $72 \mathrm{hr}$, mientras que en concentraciones inferiores, la mortalidad depende de la concentración de cobre en el medio experimental.
La mortalidad de los ejemplares mantenidos en el medio «control» fue de $6,7 \%$ al cabo de $72 \mathrm{hr}$ del ensayo. El efecto tóxico del cobre fue constante en las tres réplicas, provenientes de diferentes desoves, para cada tratamiento. Los valores obtenidos de LC50, son inversamente proporcionales al tiempo de exposición (Tabla 2, Fig. 2). Los valores de LC50 han sido altos, 660,9 ppb para $24 \mathrm{hr}$ de tratamiento y se reducen hasta valores de $212,3 \mathrm{ppb}$ a las $72 \mathrm{hr}$, es decir, hay un aumento de la sensibilidad de las larvas al medio tóxico. La declinación de los valores de LC50, que evidencia un aumento de toxicidad en función del tiempo de exposición, ha sido relativamente constante, estimándose entre 30 y $38 \%$ por día de exposición. No se evidenció gran mortalidad durante las primeras $48 \mathrm{hr}$ a concentraciones inferiores a $250 \mathrm{ppb}$ (Tabla 1).

En el tratamiento «control», los nauplii comienzan a mudar al estadio de protozoea a partir de las $72 \mathrm{hr}$ de iniciado el experimento. En los tratamientos con cobre, se evidencia, ademas de la mortalidad, un efecto sobre el comportamiento, de inhibición de la muda y malformación, especialmente en la setación de los apéndices y furca caudal, cuyas espinas no logran expandirse totalmente y son deformes. A concentraciones superiores a $125 \mathrm{ppb}$ la muda se inhibe y ninguna larva alcanza el estadio de protozoea en $96 \mathrm{hr}$ de ensayo. A concentraciones inferiores de $125 \mathrm{ppb}$ pocas larvas logran alcanzar el estadio de metanauplius o nauplius VI.

\section{DISCUSION}

Diversos experimentos de toxicidad han demostrado que los organismos acuáticos son mas sensibles al cobre que a otros iones de metales pesados (Beaumont et al., 1967; D’Agostino y Finney, 1974; Chung, 1978; Redpath, 1985). En las algas, camarones, cangrejos, moluscos bivalvos y peces, la biodisponibilidad y toxicidad del cobre puede ser reducida tanto por la presencia de iones inorgánicos como por los quelantes orgánicos hallados en aguas naturales que forman complejos con el cobre o quelantes inorgánicos agregados al agua (Sunda y Lewis, 1978; Department of Energy, 1987).

Los resultados de estas experiencias de toxicidad aguda sobre larvas nauplii de A. longinaris demuestran que la mortalidad estuvo en relación directa a la concentración del cobre y tiempo de exposición. A concentración de 1000 ppb, la mortalidad de todos los individuos se produjo durante las primeras 24 horas. En las larvas sobrevivientes, se 
observaron efectos tóxicos en alteraciones del movimiento natatorio, crecimiento larval y desarrollo. Las larvas presentaron dificultades en el desplazamiento, siendo mas lentas que en el tratamiento control y quedando totalmente inactivas en el fondo, en las altas concentraciones. También el crecimiento larval

Tabla 1. Número de ejemplares y porcentaje de mortalidad de los nauplii del camarón Artemesia longinaris empleados en las experiencias de toxicidad aguda de cobre, a concentraciones de 125, 250, 500 y 1000 ppb, durante 72 horas.

Table 1. Number of specimens and percent mortality of the shrimp Artemesia longinaris nauplii in acute toxicity tests, at concentrations of 125, 250, 500 and 1000 ppb, during 72 hours.

\begin{tabular}{|lrrrr|}
\hline \multicolumn{5}{c|}{ Tiempo (horas) } \\
Tratamiento & $\mathbf{0}$ & $\mathbf{2 4}$ & $\mathbf{4 8}$ & $\mathbf{7 2}$ \\
\hline Control & & & & \\
Total & 90 & 90 & 88 & 84 \\
$(\%)$ & 100 & 100 & 97,8 & 93,3 \\
$125 \mathrm{ppb}$ & & & & \\
Total & 90 & 90 & 87 & 73 \\
$(\%)$ & 100 & 100 & 96,7 & 81,1 \\
$250 \mathrm{ppb}$ & & & & \\
Total & 90 & 89 & 83 & 51 \\
$(\%)$ & 100 & 98,9 & 92,2 & 56,7 \\
$500 \mathrm{ppb}$ & & & & \\
Total & 90 & 87 & 19 & 4 \\
$(\%)$ & 100 & 96,7 & 21,1 & 4,4 \\
$1000 \mathrm{ppb}$ & & & & \\
Total & 90 & 0 & - & - \\
$(\%)$ & 100 & 0 & - & - \\
\hline
\end{tabular}

fue mas lento, las larvas permanecían mayor tiempo en un estadio particular sin mudar al siguiente, reduciendo el porcentaje de metamorfosis, hecho que también fue hallado en nauplius de Penaeus stylirostris a concentraciones inferiores de 0,2 uM (135 ppb) y en Metapenaeus ensis, donde a niveles de 0,06 y 0,10 mg Cu/litro (entre 80 y 100 ppb) el desarrollo larval fue significativamente mas lento que en el control (Wong et al., 1995).

Los nauplii de $A$. longinaris en los diferentes tratamientos con sulfato de cobre mostraron dificultades en desprenderse de la cutícula durante la muda, las setas y espinas no se expandían totalmente, presentando un aspecto deforme comparados con el control, características que se presentan en un medio tóxico (Scelzo, 1993). En el tratamiento control, a partir de las 72 horas de experimentación, las larvas comienzan a mudar al estadio de protozoea y todas alcanzan ese estadio a las $96 \mathrm{hr}$ de experiencia. Cuando las larvas de los crustáceos son criadas en condiciones ambientales desfavorables o en presencia de sustancias tóxicas, el crecimiento larval se reduce, presentando dificultades en la muda o ecdisis y aparecen malformaciones, especialmente a nivel de las setas furcales. Ello ha sido observado en las larvas nauplii del camarón A. longinaris, cultivada en aguas sin tratar con agentes quelantes (Scelzo, 1993 y en prensa). También se ha reportado en las larvas del camarón gigante de Malasia, Macrobrachium rosembergii criado con niveles de toxicidad aguda de nitritos (Armstrong et al., 1976). Existen ciertas afinidades y diferencias en cuanto a los valores de toxicidad hallados en estas experiencias en comparación con larvas nauplius de otras especies.

Salvando diferencias experimentales, Lawrence et al. (1981), encontraron 100\% de mortalidad en 24 horas en nauplii del camarón Penaeus stylirostris a concentraciones de 20 uM (1350,8 ppb), valores bastante similares a los hallados en el presente estudio ( $100 \%$ de mortalidad durante las primeras 24 horas a concentraciones de $1000 \mathrm{ppb}$ ) en estas experiencias. Wong et al. (1995) indican valores de cobre LC50 (48 horas) que se incrementan en relación directa con el estadio de desarrollo: $0,16 \mathrm{mg} / 1$ 


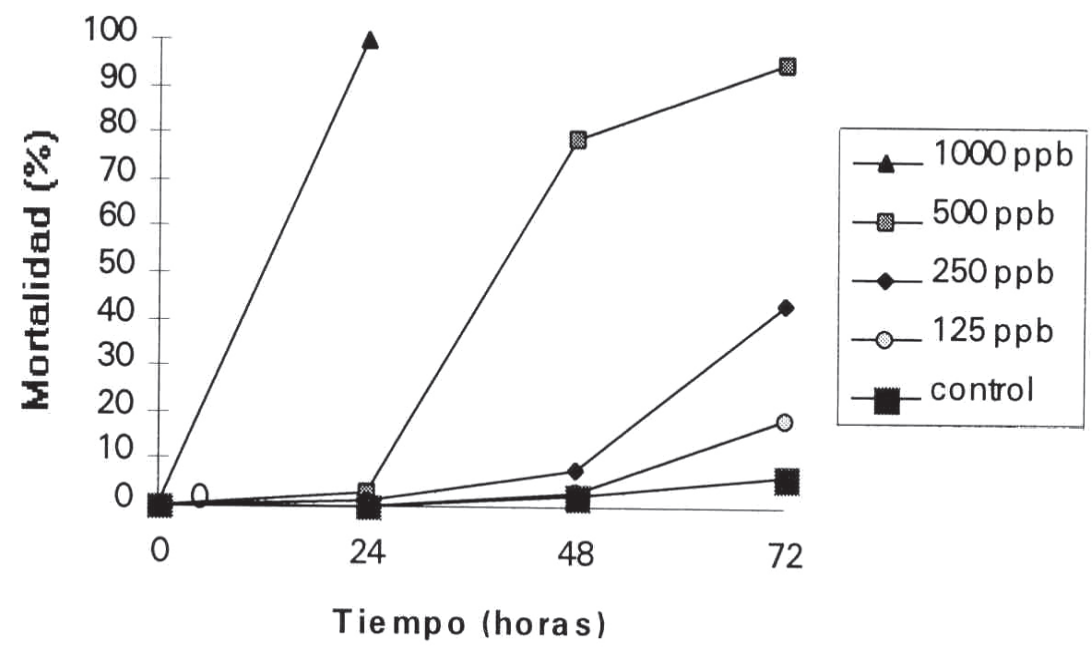

Figura 1. Mortalidad (\%) de los naupliis del camarón Artemesia longinaris en diferentes concentraciones de cobre $\left(\mathrm{CuSO}_{4}\right)$ en relación al tiempo de exposición.

Figure 1. Naupliis mortality $(\%)$ of the shrimp Artemesia longinaris at differents copper $\left(\mathrm{CuSO}_{4}\right)$ concentrations in relation to the time of expossure.

\section{Valores de LC50}

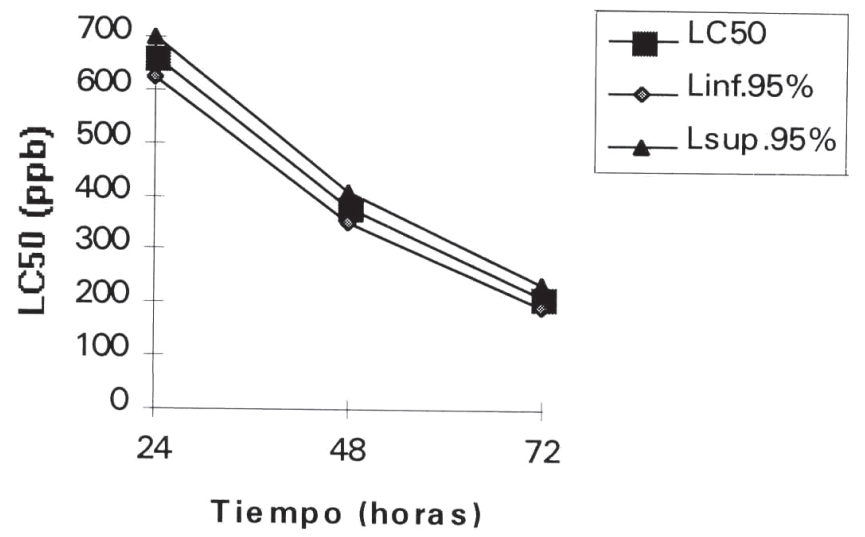

Figura 2. Valores promedios y límites de confianza de LC50 para naupliis del camarón Artemesia longinaris expuestos a diferentes concentraciones de cobre $\left(\mathrm{CuSO}_{4}\right)$.

Figure 2. Mean values and confidence limits for naupliis of the shrimp Artemesia longinaris at differents copper $\left(\mathrm{CuSO}_{4}\right)$ concentrations. 
(160 ppb) para el estadio de protozoea III, 1,58 mg/l para mysis III (1580 ppb) y 4,76 mg/l (4760 ppb) para la postlarva 3 de Metapenaeus ensis, lo cual demuestra un proceso de adaptación. Ello podría ser debido además de tratarse de especies distintas, a que fueron experiencias llevadas a cabo en condiciones ambientales diferentes (por ejemplo, temperatura). Es conocido que la absorción de cobre se incrementa en relación directa con la temperatura (Blust et al., 1994) y por otra parte, el contenido de cobre tanto en la hemolinfa como en el hapatopáncreas decrece durante el invierno, como ha sido determinado en Carcinus mediterraneus por Devescovi y Lucu (1995). También se ha demostrado que la toxicidad del cadmio y del cobre puede estar más relacionada a la actividad de los iones libres que a la concentración total de los metales (Sunda y Lewis, 1978; Department of Energy, 1987).

A nivel celular, el metabolismo de los metales como el cobre, está regulado por ligadores metálicos intracelulares específicos. Entre los grupos más prominentes de estos ligadores, están las proteínas ligadoras de metales (Metal Binding Protein $=$ MBP) de bajo peso molecular, como las proteínas similares a las metalotioneínas (Metallothionein-like proteins) (Department of Energy, 1987), tanto para el cobre (Roesijadi, 1980; Sanders et al., 1983, 1984, 1985; Jenkins, 1987), como para el mercurio (Roesijadi, 1982). Se ha determinado que una metalotioneína es un ligante de cobre muy importante en larvas de cangrejos (Jenkins, et al., 1984; Sanders y Jenkins, 1984). Muchas especies tienen habilidad de sintetizar MBP en respuesta a la exposición al metal, ligando exceso de los mismos. Un proceso que puede ser importante en la desintoxicación del cobre en mejillones, es la unión de metales a proteínas de bajo peso molecular (LMW) similares a la metalotioneínas (MT) (Harrison y Lam, 1985). Crecelius et al. (1982) demostraron que la toxicidad del cobre en larvas de camarones declinó a medida que se incrementó la abundancia de moléculas orgánicas presentes en el sedimento al formar complejos con el cobre, impidiendo la biodisponibilidad de dichos metales. Paralelamente, las bacterias bentónicas pueden resolubilizar rápidamente el sedimento que une metales (Sunda y Gillespie, 1979).

Desde el punto de vista fisiológico, las glándulas digestivas (hepatopáncreas en crustáceos) están relacionadas con el metabolismo del cobre. El transporte de los metales se realiza a través de las membranas biológicas semipermeables (branquias y otros epitelios) y debe realizarse por medio de proteínas específicas para el transporte de metales. Las membranas branquiales consisten en una variedad de proteínas funcionales embebidas en una doble capa de fosfolípidos. Estas capas poseen propiedades tanto hidrofóbicas como hidrofílicas (Nevenzel et al., 1985). Las branquias de crustáceos, de especies eurihalinas y estenohalinas, están diferenciadas generalmente, en estructura y función. La porción anterior de las branquias posteriores parecerían estar involucradas en absorción activa del sodio, control del pH sanguíneo y balance salino (Roesijadi, 1980; Benson et al., 1987; Nevenzel et al., 1985).

Dado que en las larvas no está aún desarrollado el sistema de respiración branquial, el intercambio gaseoso se produce a través de la cutícula que recubre el cuerpo. En los crustáceos, el crecimiento y la muda están íntimamente ligados entre sí, ello hace difícil las comparaciones de crecimiento entre éstos y otros organismos (Sanders et al., 1985). En las larvas de crustáceos, cuando la acumulación del cobre excede la mitad de la constante de saturación, el crecimiento larval cae rápidamente (Sanders et al., 1983). Sin embargo, frente a ciertos niveles de concentración del tóxico, inferiores a los que produciría una inhibición del crecimiento, el ritmo de crecimiento puede estar favorecido, superando al control, fenómeno conocido como «hormesis» (Sanders et al., 1985).

A nivel experimental, se emplean varias sustancias quelantes de metales, por ejemplo EDTA (ácido etilendiaminotetracético) y TRIS (Hidroximetilaminoetano), la presencia de éstos puede reducir o eliminar el efecto negativo de dichos metales (Sunda y Lewis, 1978; Lawrence et al., 1981; Cheng et al., 1987; Department of Energy, 1987). Ello justificaría el uso tan difundido de dichas sustancias en las experiencias de cría y cultivo de organismos acuáticos, garantizando buena calidad de agua, facilitando los procesos de muda en larvas de crustáceos y reduciendo la mortalidad debido a la contaminación (Scelzo, en prensa), dado que el proceso de quelación reduce la concentración de los iones de metales pesados y de esa manera, su posible toxicidad.

\section{CONCLUSIONES}

1.- El cobre, como sulfato de cobre en agua de mar (34\%o de salinidad y a temperaturas entre $18 \mathrm{y}$ $22^{\circ} \mathrm{C}$ ), produce toxicidad en larvas nauplii del camarón Artemesia longinaris, dependiendo de la concentración y tiempo de exposición. 
2.- Los estudios de toxicidad aguda demostraron que a concentraciones de cobre equivalentes a $1000 \mathrm{ppb}$, se produce mortalidad de todos los individuos sometidos a ensayo durante las primeras $24 \mathrm{hr}$. El valor de LC50-24 fue de $660 \mathrm{ppb}$, LC50-48 fue 378,5 ppb y LC50-72 fue de 212,3.

3.- Existe retardo del crecimiento en relación directa a la concentración de cobre en la solución.

\section{AGRADECIMIENTOS}

Este trabajo ha sido financiado en parte mediante subsidios del CONICET Argentina, (PID: Leg. 3-059600/ 88) y Subsidio al Proyecto 043/94 Ciclos de Vida, reproducción y cultivo experimental de crustáceos marinos, de la Univ. Nac. De Mar del Plata. Forma parte del Subprograma II. Acuicultura. Programa Iberoamericano de Ciencia y Tecnología para el Desarrollo. CYTED. El autor expresa su agradecimiento al Dr. José L. Estévez, a la Lic. Miriam Solís del CENPAT y Dra. J.E. Aizpún de Moreno UNMDP, por el asesoramiento químico y Sr. Juan C. Berón (CENPAT), quien colaboró en el desarrollo de los bioensayos.

\section{REFERENCIAS}

Armstrong, D.A., M.J. Stephenson y A.W. Knight. 1976. Acute toxicity of nitrite to larvae of the giant Malasian prawn Macrobrachium rosembergii. Aquaculture, 9: 39-46.

Beaumont, A.R., G. Tserpes y M.D. Budd. 1967. Some effects of copper on the veliger larvae of the mussel Mytilus edulis and the scallop Pecten maximus (Mollusca, Bivalvia). Mar. Environ. Res., 21: 299-309.

Blust, R., L. Van Ginneken y W. Decleir. 1994. Effeto of temperature on the uptake of copper by the brine shrim, Artemia franciscana. Aquat. Toxicol., 30: 343-356.

Boschi, E.E. y M.A. Scelzo. 1978. Desarrollo larval y cultivo del camarón comercial de Argentina Artemesia longinaris Bate (Crustacea, Decapoda, Penaeidae). In: La Acuicultura en América Latina. FAO Inf. Pesca 159(1): 287-327.

Benson, A.A., S. Chapelle, J.C. Nevenzel, J.L. Hakanson, L. Bolis y A.G. Gibbs. 1987. Environmental perturbations of gill molecular structure. In: J.V. Dorigan y F.L. Harrison (eds). Physiological Responses of Marine Organisms to Environmental Stresses, pp. 29-33.
Bryan, G.W. 1968. Concentrations of zinc and copper in the tissues of Decapod Crustaceans. J. mar. biol. Ass., U.K., 48: 303-321.

Cheng, T.C., A. Mohandas y J.T. Sullivan. 1987. In vivo effects of heavy metals on cellular immunity in molluscs. In: J.V. Dorigan y F.L. Harrison. (eds). Physiological Responses of Marine Organisms to Environmental Stresses, pp. 59-62.

Chung, K.S. 1978. Efectos letales de cadmio, cromo, cobre y zinc en Nerita fulgurans. Bol. Inst. Oceanogr. Univ. Oriente, Venezuela, 17(1-2): 31-33.

Costlow, J.D. y B.M. Sanders. 1987. Effects of cyclic temperature on larval development of marine invertebrates: II. Regulation of growth as a general indicator of stress. In: J.V. Dorigan y F.L. Harrison (eds). Physiological Responses of Marine Organisms to Environmental Stresses, pp. 105-109.

Crecelius, E.A. y N.S. Bloom. 1987. Marine chemistry of energy-related contaminants. In: J.V. Dorigan y F.L. Harrison (eds). Physiological responses of marine organisms to environmental stresses, pp. 121-125.

Crecelius, E.A., J.T. Hardy, G.I. Gibson, R.L. Schmidt, C.W. Apts, J.M. Gurtisen y S.P. Joyce. 1982. Copper bioavailability to marine bivalves and shrimp: Relationship to cupric ion activity. Mar. Environ. Res., 6: 13-26.

D'Agostino, A. y C. Finney. 1974. The effect of copper and cadmiun on the development of Tigriopus japonicus. In: F.J. Vernberg and W.B. Vernberg (eds). Academic Press. Pollution and Physiology of Marine Organisms, pp. 445-463.

Department of Energy, USA. 1987. Physiological responses of marine organisms to environmental stresses. Dorigan, J.V. and F.L. Harrison (eds). DOE/ER-0317: 501 pp.

Devescovi, M. y C. Lucu. 1995. Seasonal changes of the copper level in shore crabs Carcinus mediterraneus. Mar. Ecol. Progr. Ser., 120: 169-174.

Harrison, F.L. y J.R. Lam. 1985. Partitioning of copper among copper-binding proteins in the mussel Mytilus edulis exposed to soluble copper. Mar. Environ. Res., 16: 151-163. 
Iorio, M.I., M.A. Scelzo y E.E. Boschi. 1990. Desarrollo larval y postlarval del langostino Pleoticus muelleri Bate, 1888 (Crustacea, Decapoda, Solenoceridae). Sci. Mar., 54(45): 329-341.

Jenkins, K.D. 1987. Metal metabolisms in marine organisms: mechanisms and limits. In: J.V. Dorigan y F.L. Harrison (eds). Physiological Responses of Marine Organisms to Environmental Stresses, pp. 15: 311-313.

Jenkins, K.D., B.M. Sanders y J.D. Costlow. 1984. Regulation of copper accumulation and subcellular distribution in developing crab larvae: The role of metallothionein and other intracellular Cu-binding ligands. Mar. Environ. Res., 14: 474-475.

Laws, E.A. 1981. Aquatic Pollution. An Introductory Text. J. Wiley and Sons. N.Y.

Lawrence, A.L., J. Fox y F.L. Castille, Jr. 1981. Decreased toxicity of copper and manganese ions to shrimp nauplii (Penaeus stylirostris Stimpson) in the presence of EDTA. J. World Maricult. Soc. 12(1): 271-280.

Lichtfield, J.T. y F. Wilcoxon. 1949. A simplified methods of evaluation dose-effect experiments. J. Pharmacol. Exp. Therapeutics, 96: 99-113.

Nevenzel, J.C., A. Gibbs y A.A. Benson. 1985. Plasmologens in the gill lipids of aquatic animals. Comp. Biochem. Physiol., 82B(2): 292-297.

Porta, A.A. 1991. Especiación del Cu (II) en sistemas acuáticos. Efecto de la presencia de complejantes naturales. Tesis Doctoral, Universidad Nacional de La Plata.

Redpath, J.K. 1985. Growth inhibition and recovery in mussels (Mytilus edulis) exposed to low copper concentrations. J. mar. biol. Ass. U.K., 158: 233-247.

Roesijadi, G. 1980. Influence of copper on the clam Protothaca staminea: effects on gills and occurrence of copper-binding proteins. Biol. Bull., 158: 233-247.

Roesijadi, G. 1982. Uptake and incorporation of mercury into mercury-binding proteins of gills of Mytilus edulis as a function of time. Mar. Biol., 66: 151-157.
Sanders, B., J.D. Costlow, K.D. Jenkins y T. Marshall. 1984. The effects of varying cupric ion activities on copper uptake and cytosolic distribution and growth in crab larvae. Mar. Environ. Res., 14: 476-477.

Sanders, B. y K.D. Jenkins. 1984. Relationships between free cupric ion concentration in sea water and copper metabolisms and growth in crab larvae. Biol. Bull., 167: 704-712.

Sanders, B., K.D. Jenkins, W.G. Sunda y J.D. Costlow. 1983. Free cupric ion activity in seawater: effects on metallothionein and growth in crab larvae. Science, 222: 53-55.

Sanders, B., R.B. Laughlin y J.D. Costlow. 1985. Growth regulation in larvae of the mud crab Rhitropanopeus harrisii. In: A.M. Wenner (ed.). Crustacean issues: 155-161. A.A. Balkema Press, Rotterdam.

Scelzo, M.A. 1987. Posibilidades del cultivo de camarones y langostinos marinos en Argentina. In: Panorama de la Acuicultura en la Argentina. SECYT. Subsecretaría de Coordinación y Planificación. Delegación Regional Patagonia. CRUB-Univ. Nac. Comahue, Cuadernos Universitarios, 17: 23-34.

Scelzo, M.A. 1993. Malformaciones en larvas del camarón Artemesia longinaris: efectos subletales frente a contaminación y baja calidad de las aguas de los cultivos. In: Seminario Internacional Acuicultura y Medio Ambiente, Fundación Chile, Santiago. Chile, Resúmenes de Posters: pp. 1-2.

Scelzo, M.A. Efecto del EDTA. (Acido Etilendiaminotetracético) en desoves del camarón Artemesia longinaris (Crustacea, Decapoda, Penaeidae). Frente Marítimo, (en prensa).

Scelzo, M.A., N. Lucero y M. Giangiobbe. (MS). Toxicidad aguda del nitrito en ejemplares juveniles y preadultos del camarón Artemesia longinaris (Decapoda, Penaeidae). Enviado a: Nauplius, Brasil.

Sprague, J.B. 1969. Measurement of pollutant toxicity to fish. I. Biossay methods for acute toxicity. Review paper. Water Res., 3: 793-821.

Sunda, W.G. y P.A. Gillespie. 1979. The response of a marine bacterium to cupric ion and its use 
to estimate cupricion activity in seawater. J. Mar. Res., 37(4): 511-529.

Sunda, W.G. y G.A.M. Lewis. 1978. Effect of complexation by natural organic ligands on the toxicity of copper to a unicellular algae, Monochrysis lutheri. Limnol. Oceanogr., 23(5): 870-876.

Waldichuk, M. 1974. Some biological concern in heavy metals pollution. In: Vernberg, F.J. and W. Vernberg (eds). Pollution and Physiology of Marine Organisms. Academic Press, N.Y.; pp. $1-59$.

Recibido el 23 de julio de 1996.

Aceptado el 29 de diciembre de 1996.
Water Pollution Control Federation. 1980. Standard methods for the examination of water and wastewater. Fifteen Ed. American Publica Health Ass. Washington.

Wong, C.K.,J.K. Cheung y K.H Chu. 1995. Effects of copper on survival, development and growth of Metapenaeus ensis larvae and postlarvae (Decapoda, Penaidae). Mar. Pollut. Bull:, 31(4-12): 416-419. 